\title{
Implante cirúrgico do balão intra- aórtico: uso de uma nova técnica
}

\author{
Rui Manoel de Souza Sequeira A. de ALMEIDA*, Maria Joāo Amorim FERREIRA*, \\ Edison José RIBEIRO*, Danton R. da Rocha LOURES*
}

RBCCV $44205-312$

Almeida R M S S, Ferreira M J A, Ribeiro E J, Loures D R R - implante cirúrgico do balăo intra-aórtico: uso de uma nova técnica. Rev Bras Cir Cardiovasc 1996; 11 (3):216-9.

RESUMO: É descrita uma nova técnica para contrapulsação intra-aórtica, por dissecçăo cirúrgica da artéria femoral, e introdução do balão intra-aórtico através de um segmento de veia safena. A remoçāo do balăo evita a realizaçâo de nova incisăo cirúrgica, diminui as causas de obstruçăo das artérias e a infecçăo de ferida cirúrgica. Fol estudada a evoluçăo de 18 pacientes operados.

DESCRITORES: Balão intra-aórtico, métodos. Balăo intra-aórtico, efeitos. Artéria femoral,cirurgia. Veia safena, cirurgia. Contrapulsaçăo, aórtica. Circulaçăo assistida, métodos.

\section{INTRODUÇÄO}

Com os estudos experimentais de MOULOPOLOUS et al. (1) e CLAUSS et al. (2), iniciou-se o conceito da contrapulsaçăo aórtica. $O$ uso clínico da assistência circulatória com balāo intra-aórtico (BIA) foi iniciado nos anos sessenta com os trabalhos de KANTROWITZ et al. (3). Porém, foi somente nos anos setenta, que começaram a aparecer estudos sobre BIA com número apreciável de pacientes (4). Atualmente, $2 \%$ a $12 \%$ dos pacientes submetidos a cirurgia cardíaca fazem uso de BIA, com mortalidade que pode variar de $34 \%$ a $56 \%$ (5). Com o aumento das indicações ${ }^{(6)}$ e o crescimento da cirurgia cardiaca, vários autores ${ }^{(7-12)}$ referem bons resultados, em pacientes que fizeram uso deste tipo de suporte circulatório, tornando-se o método de suporte circulatório mais utilizado.

Em 1980, BREGMAN et al. (13) e SUBRAMANIAN et al. (14) descreveram a introdução do cateter-balão através da punção percutânea da artéria femoral, pela técnica de SELDINGER (15).
Apesar do método de inserçăo percutânea ser, em principio, mais fácil e rápido, revisões da literatura mostram um indice de complicaçöes vasculares, que podem chegar a $42,5 \%$ (20). A colocação do balăo por dissecção, quer seja pela introdução na aorta ou na artéria femoral, tem sido mais utilizada em cirurgia cardíaca (16); a introdução pela região inguinal é mais comum em operaçōes de revascularizaçāo do miocárdio, nas quais o acesso à artéria é feito pela mesma incisāo da abordagem da veia safena.

Este trabalho tem como finalidade descrever uma nova técnica, iniciada pelo autor (Rui Manoel de Almeida), para a inserçăo cirúrgica do cateterbalāo e sua retirada, bem como apresentar a experiência clínica inicial.

\section{CASUISTICA E MÉTODOS}

Com o paciente sob anestesia geral, a artéria femoral comum esquerda é dissecada e reparada

\footnotetext{
Trabalho realizado nos Serviços de Cirurgia Cardiovascular do Instituto de Moléstias Cardiovasculares de Cascavel e do Hospital Evangélico de Curitiba, PR, Brasil.

"Do Instituto de Moléstias Cardiovasculares de Cascavel e do Hospital Evangelico de Curitiba.

Recebido para publicaçäo em julho de 1996.

Endereço para correspondencia: Rui Manuel Sequeira de Almeida. Instituto de Moléstias Cardiovasculares de Cascavel. Policlinica de Cascavel. Rua Maranhão, 945. Cascavel, PR, Brasil. CEP 85.806-050.
} 
na porção proximal, bem como as porçōes proximais das artérias femorais profunda e superficial. Após heparinização do paciente e pinçamento das três artérias femorais, é realizada uma incisăo transversal, na parede anterior da femoral comum, compreendendo $1 / 4$ da circunferência do vaso. Esta aber. tura poderá variar, em decorrência do diâmetro da artéria, sem comprometer a introdução do cateterbalāo e evitar seqũelas na parede do vaso. Utilizase um segmento de veia safena do paciente, ou homóloga, de aproximadamente 5 centímetros de comprimento, por onde é introduzido o cateter-balão $\Theta$, em seguida, na incisăo da artéria femoral. O segmento da veia safena é colocado sobre a artéria e anastomosado com uma sutura contínua, de fio 6-0 de Polipropileno. A porçăo posterior da anastomose deverá ser realizada inicialmente, pela sua dificuldade técnica. A traçăo do balão e a não total aproximação veia/artéria permite melhor visão da linha de sutura. Esta deve ser feita com extremo cuidado, para evitar solução de continuidade na camada intima da artéria. A veia safena é amarrada ao cateter, na sua porção distal, com fio de seda 2 (Figura 1). O cateter-balão é fixado na pele, nos locais próprios, com a fixação da capa do balāo. A incisão é fechada por planos anatômicos, sendo a sutura da pele realizada com pontos separados.

A retirada do cateter-balão é feita sob anestesia local, sendo a veia tracionada até a visibilizaçăo do fio de seda; este é secionado e o cateter-balăo retirado. A veia safena é novamente ligada 1 a 2 centimetros abaixo do local onde estava amarrado - fio de seda e a sua porção distal secionada; assim, a veia retraí-se, novamente, através da sutura da pele, para o interior da incisão.

Entre abril de 1992 e maio de 1995, foram operados nos Serviços de Cirurgia Cardiovascular do Instituto de Moléstias Cardiovasculares de Cascavel e do Hospital Evangélico de Curitiba, 1139 pacientes. Destes, $5,6 \%$ necessitaram de assistência circulatória com 0 uso do balăo intra-aórtico.

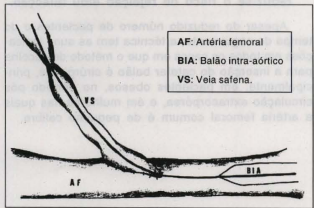

Fig.1 - Aspecto final do enxerto de vela safena na artéria femoral.
Foi utilizado o balăo intra-aórtico manufaturado pela Datascope Corporation. A técnica acima descrita foi usada em $18(28,6 \%)$ pacientes com média de idade de 57,3 anos, sendo que $55,6 \%$ eram do sexo masculino. Em todos os casos o cateter-balão foi introduzido no ínicio da operação, antes da colocação do paciente em circulaçăo extracorpórea, sendo, porém, somente iniciado o suporte circulatório na fase final.

\section{RESULTADOS}

Os pacientes permaneceram, em média, 18,8 horas utilizando o BIA. Após a retirada, não houve complicação grave, sendo o pulso pedioso palpável em 17 pacientes e detectável com Doppler em 1. Em 1 paciente houve parestesia do membro inferior esquerdo, com retorno à normalidade após 12 dias, provavelmente em decorrência da dissecção da artéria femoral, com eventual lesão do nervo femoral (Tabela 1).

No pós-operatório imediato (30 dias) não houve intercorrência e/ou complicaçāo relacionada à técnica de inserção e retirada do balão, como isquemia do membro inferior ou infeç̧āo local. A mortalidade cirúrgica foi de $33,3 \%$, sem relação com a técnica apresentada (Tabela 1).

\section{COMENTÁRIOS}

Desde a sua aplicação clínica, ○ BIA tem sido reconhecido como um método de assistência circulatória (contrapulsação) de fácil manuseio e de eficácia comprovada. A sobrevida dos pacientes tem aumentado, já que este método produz um aumento da perfusão coronária na diástole, aumentando a oferta de oxigênio ao miocárdio, uma redução do consumo miocárdico de oxigênio, pela diminuiçăo da pós-carga e tensão da parede ventricular esquerda, e um aumento do débito cardíaco.

Cerca de $2 \%$ a $12 \%$ dos pacientes submetidos a cirurgia cardiaca necessitam de assisténcia circulatória com BIA $(5,8)$. Nos Serviços de Cirurgia Cardiovascular do Instituto de Moléstias Cardiovasculares de Cascavel e do Hospital Evangélico de Curitiba, a porcentagem é semelhante, tendo-se utilizado a assistência circulatória com BIA em 5,6\% dos casos.

Com um número tão elevado de pacientes têm sido várias as técnicas usadas para a inserção e a remoção do BIA. Todas têm as suas vantagens $e$ desvantagens. No entanto, o grande divisor fica entre a punção percutânea da artéria femoral e os métodos cirúrgicos de inserção. Entre estes, devem 
Almeida RM SS, Ferreira M J A, Ribeiro EJ, Loures D R R - Implante cirúrgico do balão intra-aórtico: uso de uma nova técnica. Rev Bras Cir Cardiovasc 1996;11 (3):216-9.

TABELA 1

TIPO DE CIRURGIA, TEMPO DE BIA E COMPLICAÇÕES

\begin{tabular}{|c|c|c|c|c|}
\hline$N^{2}$ & CIRURGIA & TEMPO BIA & OBS & MORTALIDADE \\
\hline 1 & R.C. $c / T I E ~ p / R I A+S a f p / C D E M g C x$ & $2 \mathrm{~h}$ & & \\
\hline 2 & Troca V.Ao.p/P.B. n 23 & $4 h$ & & \\
\hline 3 & Troca V.Ao p/ P.B. n 23 + Ampliaçāo Anel Aórtico & $20 \mathrm{~h}$ & & \\
\hline 4 & R.C. c/Saf. p/ RIA O MgCx & $20 n$ & & \\
\hline 5 & Retroca V.AO. p/P.B n 25 & $40 h$ & Dim. Pulso & \\
\hline 6 & R.C. c/ Saf. p/ RIA sem CEC & $6 h$ & & \\
\hline 7 & R.C. c/ Saf. p/ RIA e MgCx & $24 \mathrm{~h}$ & & \\
\hline 8 & Troca V.Ao.p/ P.B. $n^{2} 23$ & $18 \mathrm{~h}$ & & Obito \\
\hline 9 & R.C. c/ Saf. p/RIA & $10 \mathrm{~h}$ & & Obito \\
\hline 10 & Troca V.Mi. p/P.B. $n^{2} 29$ + Fechamento D.S.A & $4 \mathrm{~h}$ & & Obito \\
\hline 11 & Troca V.Mi. p/ P.B. $n^{\circ} 29$ & $6 \mathrm{~h}$ & & \\
\hline 12 & Troca V. Mi. p/P.B. $n^{2} 31$ & $30 \mathrm{~h}$ & & Obito \\
\hline 13 & R.C. $c /$ Saf, $p /$ RIA, CD, $D g \in M g C x$ & $12 \mathrm{~h}$ & & Obito \\
\hline 14 & R.C. c/ MIE p/ RIA e Sat. CD e MgCx & $6 h$ & & \\
\hline 15 & Troca V.Mi p/ P.B. $n^{\circ} 31$ & $20 \mathrm{~h}$ & Parestesia & \\
\hline 16 & R.C. c/ MIE p/ RIA e Saf. Dg & $2 \mathrm{~h}$ & & \\
\hline 17 & Aneurismectomia + R.C, c/ Saf. p/CD e Dg & $63 \mathrm{~h}$ & & \\
\hline 18 & Troca V.Mi. p/P.B. $n^{2} 29+$ V.Ao p/P.B. $n^{2} 25$ & $70 \mathrm{~h}$ & & Obito \\
\hline
\end{tabular}

CD: Artéria coronária direita; CEC: Circulaçăo extracorpórea; Dg: Artéria coronária ramo diagonal; DSA: Defeito do septo atrial MgCx: Artéria coronária ramo marginal da circunflexa; P.B.: Prótese de pericárdio bovino; RIA: Artéria coronária ramo interventricular anterior; R.C.: Revascularização coronariana; Saf: Veia safena; TIE: Artéria torácica interna esquerda; V.Ao: Valva aórtica; V.Mi. Valva mitral.

salientar-se a técnica da bolsa (17) ou sutura (9) e a incisão longitudinal ou transversal da artéria com o uso de um segmento de tubo Dacron (18).

Vários autores apresentam porcentuais de complicaçōes semelhantes entre a inserçāo por punçāo (percutânea ou "aberta") e a cirurgia $(10,19,20)$. No entanto, parece haver uma tendência para se usar a inserçăo cirúrgica, quando o paciente está no centro cirúrgico e/ou é feita pelo cirurgião. Nestes casos, a taxa de complicações em decorrência da inserção e da retirada parece ser menor (21).

As complicaçōes mais freqüentes, tanto na inserçăo como na retirada do balão, săo vasculares. No entanto, vários săo os fatores que podem atuar, como a experiência do cirurgiāo, o local (centro cirúrgico ou unidade de terapia intensiva) e a necessidade de um procedimento rápido (22).

A técnica apresentada tem algumas vantagens, principalmente no que se refere à retirada do balăo:

1) não há necessidade de uma nova operaçāo, podendo mesmo ser realizada no leito;

2) năo se faz uso de material protético, como
Dacron, que tem de ser excluído na retirada do balão;

3) a linha de sutura entre a artéria femoral e a veia safena (como nas anastomoses aorto/ safena na revascularização miocárdica) não produz turbulência do fluxo normal da artéria femoral, nāo havendo, portanto, necessidade da retirada da vela;

4) a veia tende a trombosar sem a formação de trombos na luz da artéria, fato já estabelecido na oclusão dos enxertos de veia safena, nas operações de revascularizaçăo do miocárdio;

5) usando-se veia do paciente ou homóloga reduz-se o risco de rejeição e/ou infecção.

Apesar do reduzido número de pacientes e do tempo de seguimento, a técnica tem as suas indicaçōes em todos os casos em que o método de escolha para a inserçăo do cateter balăo é cirúrgico e, principalmente, em pacientes obesos, no periodo pós circulação extracorpórea, e em mulheres nas quais a artéria femoral comum é de pequeno calibre. 
RBCCV $44205-312$

Almeida R M S S, Ferreira M J A, Ribeiro E J, Loures D R R - Surgical insertion of intra-aortic balloon: a new technique. Rev Bras Cir Cardiovasc 1996; 11 (3):216-9.

ABSTRACT: This paper describes a new technique for insertion and removal of intra-aortique balloon trough a segment of saphenous vein. The balloon's removal is accomplished without a new surgical intervention, which decreases many complications. The authors studied the evolution of 18 patients operated on with this new technique.

DESCRIPTORS: Intra-aortic balloon, methods. Intra-aortic balloon, effects. Femoral artery, surgery. Saphenous vein, surgery. Counterpulsation, aortic. Assisted circulation, methods.

\section{REFERÊNCIAS BIBLIOGRÁFICAS}

1 Moulopolous S D, Topaz W, Kolpp W J-Diastolic balloon pumping (with carbon dioxide) in the aorta: a mechanical assistance to failing circulation. Am Heart $J$ 1962; 63: 669-75.

2 Clauss R M, Missier P, Reed G F, Tice D - Assisted circulation by counterpulsation with an intra-aortic balloon: methods and affects. In: Digest. 15 th Annual Conference on Engineering in Medicine and Biology. Chicago: Northwestern University, 1962: 44.

3 Kantrowitz A, Akutsu T, Chaptal P A, Krakauer J, James R T - A clinical experience with an implanted mechanical auxiliary ventricle. JAMA 1966; 197 . 525-9.

4 Scheidt S, Wilner G, Mueller $\mathrm{H}$ et al. - Intra-aortic balloon counterpulsation in cardiogenic shock: report of a cooperative clinical trial. N Engl J Med 1973; 288: 979-84.

5 Naunheim K S, Swartz M T, Pennington D G - Intraaortic balloon pumping in patients requiring cardiac operations: risk analysis and longterm follow-up. $J$ Thorac Cardiovasc Surg 1992; 104: 1654-61.

6 Figulla H R - Circulatory support devices in clinical cardiology: current concepts. Cardiology 1994: 84: 149-55.

7 Corral C H \& Vaughn C C - Intra-aortic balloon counterpulsation: an eleven-year review and analysis of determinants of survival. Texas Heart Inst $J$ 1986; 13: 39-44.

8 Di Lello F, Mullen D C, Flemma R J, Anderson A J. Kleinman L H - Results of intra-aortic balloon pumping after cardiac surgery: experience with the Percor balloon catheter. Ann Thorac Surg 1988; 46:442-6.

9 Goldman B S, Hill T J, Rosenthal G A, Scully H E, Weisel R D. Baird R J - Complications associated with use of the intra-aortic balloon pump. Can J Surg 1982; 25: 153-6.

10 Hauser A M, Gordon S, Gangadharan V et al. Percutaneous intra-aortic balloon counterpulsation: clinical effectiveness and hazards. Chest 1982; 82 422-5.

11 Michels $R$, Haalebos $M$, Kint $P$ P, Hagemeijer $F$,
Balkumaran K, Van Der Brand M - Intra-aortic balloon pumping in myocardial infarction and unstable angina. Eur Heart $J$ 1980; 1: 31-43.

12 Sturn J T, McGee M G, Fuhrman T M et al. - Treatment of postoperative low output syndrome with intra-aortic balloon pumping experience with 419 patients. Am $J$ Cardiol 1980; 45: 1033-6.

13 Bregman D, Nichols A B, Weiss M B, Powers E R, Martin E C, Cassarela W J - Percutaneous intra-aortic balloon insertion. Am J Cardiol 1980; 46: 261-7.

14 Subramanian V A, Goldstein J E, Sos T A, McCabe J C. Hoover E A, Gay W A - Preliminary clinical experience with percutaneous intra-aortic balloon pumping. Circulation 1980; 62 (Suppl 1): 123-9.

15 Seldinger S I - Catheter replacement of the needle in percutaneous arteriography: a new technique. Acta Radiol (Stockholm) 1953; 39: 368-70.

16 Robicsek $F$, Masters $T$ N, Rice $H$, Morency R P Enhancing the applicability and effectiveness of intraaortic balloon counterpulsation. J Card Surg 1990; 5: $321-7$.

17 Zada F, McCabe J C, Subramanian V A - Simplified technique for intra-aortic balloon insertion. Ann Thorac Surg 1980; 29: 573-4.

18 Payne D D, Sewell D $H$, Amato $J \mathrm{~J}$, Faraci $P$ A, Rheinlander H F, Cleveland R J - Transverse femoral arteriotomy for intra-aortic balloon insertion. Ann Thorac Surg 1978; 26: 263-5.

19 Cutler B S, Okike N O, Salm T J V - Surgical versus pencutaneous removal of the intra-aortic balloon. $J$ Thorac Cardiovasc Surg 1983; 86: 907-11.

20 Yuen J C - Percutaneous intra-aortic balloon pump: emphasis on complications. South Mod J 1991; 84: 956-60.

21 Rohrer M J, Sullivan C A, McLaughlin D J, Cutler B S . A prospective randomized study comparing surgical and percutaneous removal of intra-aortic balloon pump. J Thorac Cardiovasc Surg 1992; 103: 569-72.

22 Shahian D M, Neptune W B, Ellis Jr F H, Maggs P R . Intra-aortic balloon pump morbidity: a comparative analysis of risck factors between percutaneous and surgical techniques. Ann Thorac Surg 1983;36: 644-53. 